\title{
Prediction Efficiency of Postoperative Acute Kidney Injury in Acute Stanford Type A Aortic Dissection Patients with Renal Resistive Index and Semiquantitative Color Doppler
}

\author{
Huai Qin, ${ }^{1}$ Yaqiong $L i,{ }^{2}$ Nan Zhang, ${ }^{3}$ Tiezhu Wang, ${ }^{4}$ and Zhanming Fan ${ }^{3}{ }^{3}$ \\ ${ }^{1}$ Department of Ultrasound, Beijing Anzhen Hospital, Capital Medical University, \\ Beijing Institute of Heart Lung and Blood Vessel Diseases, Beijing, China \\ ${ }^{2}$ Department of Cardiovascular Intensive Care, Beijing Anzhen Hospital, Capital Medical University, \\ Beijing Institute of Heart Lung and Blood Vessel Diseases, Beijing Aortic Disease Center, Beijing, China \\ ${ }^{3}$ Department of Radiology, Beijing Anzhen Hospital, Capital Medical University, \\ Beijing Institute of Heart Lung and Blood Vessel Diseases, Beijing, China \\ ${ }^{4}$ Department of Ultrasound, Hospital of Renmin University of China, Beijing, China
}

Correspondence should be addressed to Zhanming Fan; fanzm120@126.com

Received 11 June 2019; Accepted 23 September 2019; Published 3 December 2019

Guest Editor: Qian Fan

Copyright (c) 2019 Huai Qin et al. This is an open access article distributed under the Creative Commons Attribution License, which permits unrestricted use, distribution, and reproduction in any medium, provided the original work is properly cited.

Objectives. This study is aimed to evaluate the efficiency in early prediction of postoperative persistent acute kidney injury (PAKI) after surgery in acute Stanford type A aortic dissection (AAAD) patients by using Doppler renal resistive index (RRI) and semiquantitative color (SQC) Doppler grade, respectively. Methods. 84 AAAD patients received Sun's surgical management, and 67 patients were enrolled. RRI and SQC Doppler grade were evaluated by ultrasonography, respectively, at 6 hours after surgery. Serum creatinine (sCr) was recorded before operation and at 24 hours, 48 hours, and 72 hours after operation. AKI grade was evaluated according to the classifications of the Acute Kidney Injury Network (AKIN). PAKI is defined as persistent oliguria and/or sCr elevation after 3 days. RRI and SQC Doppler grade were compared, respectively, between the PAKI and non-PAKI groups. Potential predictors were first tested by univariate logistic regression analysis, and a multivariate model was identified to determine the independent predictive ability of RRI and SQC Doppler grade for the PAKI. Receiver operating characteristic (ROC) analysis was performed to compare the diagnostic accuracy between RRI and SQC Doppler grade in early prediction of PAKI by using AKIN classifications as the reference standard. Results. Of a total of 67 patients enrolled during the study period, 21 (31.3\%) patients suffered from PAKI and 8 (11.9\%) patients required dialysis. There are significant differences in RRI $(0.80 \pm 0.09$ vs. $0.70 \pm 0.05, P=0.002)$ and SQC Doppler grade $\left(x^{2}=12.193\right.$, $P=0.007$ ) between the 2 groups with and without PAKI. Univariate analysis showed that RRI, SQC Doppler grade, length of stay in ICU, time of CPB, and length of stay in hospital were significant predictors of PAKI. RRI and the SQC Doppler grade remained independent predictors of PAKI. Area under the curve (AUC) of RRI was 0.855 (95\% CI, 0.74-0.96) with cutoff value 0.725 (sensitivity 90.9\% and specificity 71.1\%), AUC of SQC Doppler grade was 0.642 (95\% CI, $0.49-0.79)$ with cutoff value grade 2 (sensitivity $50 \%$ and specificity 73.3\%). Conclusion. Both postoperative RRI and SQC Doppler grade are independent predictors for PAKI after surgery in AAAD patients. Both postoperative RRI and SQC Doppler grade can be obtained rapidly by bedside ultrasound, which is a good tool for early prediction for postoperative PAKI.

\section{Introduction}

The gold standard for the diagnosis of postoperative AKI is serum creatinine (sCr), but it is not used for early detection of AKI $[1,2]$. Recent studies provide early diagnosis of AKI with several urine and blood markers [3-6]. Commonly used urine markers included neutrophil gelatinase-associated lipocalin (NGAL), cystatin C, kidney injury molecule 1 (KIM-1), interleukin-18 (IL-18), liver-type fatty acid-binding protein (L-FABP), and other more. Blood markers include cystatin $\mathrm{C}$, uric acid, and monocyte chemoattractant protein-1 (MCP-1). Although these indicators have certain 
efficacy while intraoperative monitoring, urine and blood markers cannot be used to predict severe AKI [7].

Postoperative AKI is an ischemia-reperfusion injury, so renal perfusion is associated with AKI [8]. There are several imaging methods to evaluate renal perfusion. Computed tomography angiography (CTA) can evaluate renal perfusion, but the postcontrast AKI carries a risk of more permanent renal insufficiency $[9,10]$, which is also not suitable for daily bedside operation. Isotope renogram is a very sensitive, simple, and noninvasive method for evaluating renal parenchymal function [11], but it requires the patient to go to the fixed examination room and is also not suitable for the critically ill patients in the postoperative care unit.

Ultrasound is being used increasingly, especially in the intensive care unit (ICU) after operation, which has been recognized by the ICU doctors. The RRI measured by Doppler can be worked as a marker to predict AKI in some kinds of cardiac operations [12-15]. However, some other literatures do not consider RRI to be a good indicator of renal perfusion $[16,17]$. RRI as a marker to predict AKI needs to be confirmed in more research studies. Moreover, intrarenal arcuate or interlobar arteries cannot be displayed when critical AKI occurred, RRI was hardly to be obtained, and the clinical application was limited. The color Doppler can intuitively and instantly observe renal perfusion, and semiquantitative evaluation can be acquired rather than an accurately quantitative renal perfusion assessment [18]. But few studies about this indicator are reported. The purpose of this study was to evaluate efficiency of RRI and SQC Doppler for prediction of PAKI after surgery in AAAD patients.

\section{Patients and Methods}

This study was conducted in accordance with the Declaration of Helsinki. This study was approved by the Ethics Committees of Beijing Anzhen Hospital, Capital Medical University. The committee agreed with using the data obtained from the postoperative patients in the ICU.

2.1. Patients. A prospective, observational study was performed during a nonconsecutive period (September 2017March 2018) at Beijing Anzhen Hospital, cardiac surgery intensive care unit. Inclusion criteria were as follows: the AAAD patients received Sun's surgical operation with age $>18$ years and inclusion within 24 hours after ICU admission. Exclusion criteria were as follows: (1) history of chronic kidney dysfunction and preoperative renal injury; (2) pregnancy; (3) death during 72 hours after operation; (4) kidney tumor $(n=1)$; (5) known renal artery stenosis; (6) arrhythmia; and (7) incomplete data. 84 AAAD patients received Sun's surgical operation, and 67 patients were analyzed (Figure 1), including 53 males and 14 females.

2.2. SQC Doppler Grade Evaluation. All ultrasound measurements and evaluations were performed using a $3-5 \mathrm{MHz}$ pulsed wave Doppler probes with GE equipment (Vivid E9, GE Healthcare, Horton, Norway) by one of the investigators. The kidneys were displayed along a longitudinal section with

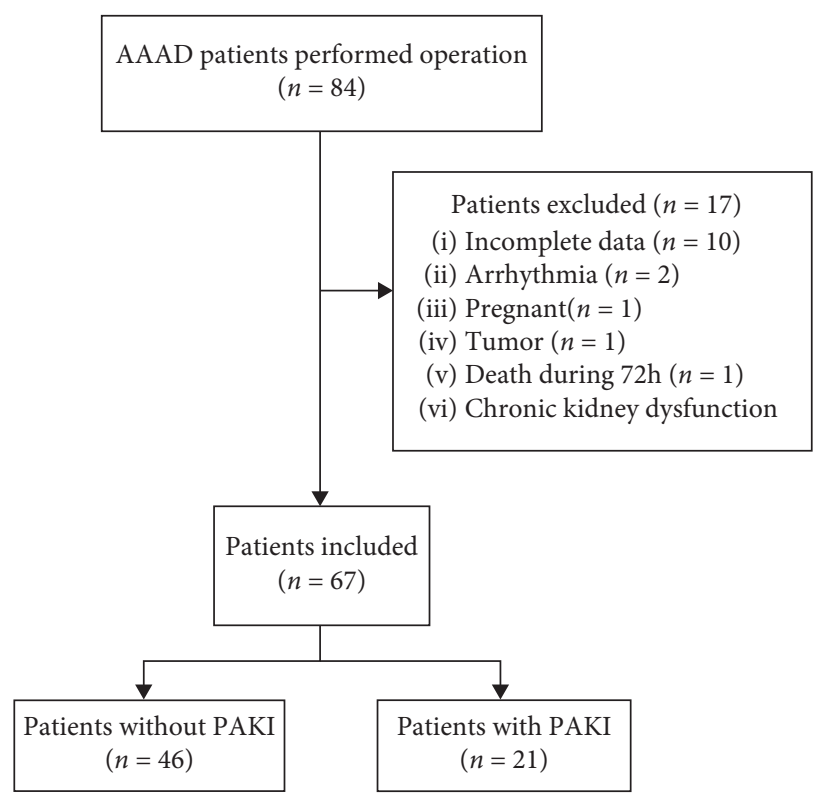

FIgURE 1: Flowchart of the study (AAAD: acute Stanford type A aortic dissection; PAKI: persistent acute kidney injury).

two-dimensional ultrasound at the renal hilum slice. The color Doppler gain was set to minimal background noise. Each renal perfusion was evaluated by color Doppler using the semiquantitative scale (Table 1 and Figure 2) [19].

As the perfusion in the bilateral kidney is diverse in AAAD patients, the renal perfusion grade was defined as the average.

2.3. RRI Measurement. Renal blood flow was located along a longitudinal section showing the renal hilum [18] and interlobar arteries. Sampling any side midinterlobar artery (IRA) with an angle of blood stream $<30^{\circ}$. Set to minimal background noise, the Doppler gain can obtain at least three similar continuous waveforms. In each kidney, interlobar artery peak systolic velocity and end diastolic velocity were measured three times at the Doppler spectrum. RRI was calculated by the equation as follows:

$$
\text { RRI }=\frac{\text { (peak systolic velocity }- \text { end diastolic velocity) }}{\text { peak systolic velocity }} \text {. }
$$

Because of diversity at the bilateral kidneys perfusion in AAAD patients, RRI was recorded as average.

2.4. Study Design. Ultrasound SQC Doppler grade and RRI measurements were performed at 6 hours after surgery.

We started to measure the RRI and SQC Doppler grade of 14 kidneys in 7 patients by a double-blind method, in order to compare the consistency of the two operators.

$\mathrm{sCr}$ was recorded before operation (creatinine was defined as the last known creatinine value before operation) and 24 hours, 48 hours, and 72 hours after operation. AKI was classified into three grades according to the AKIN classification system [20]. PAKI was defined as persistent 
TABLe 1: Semiquantitative color Doppler scale in renal perfusion.

\begin{tabular}{|c|c|}
\hline Grade 0 & Unidentifiable vessels \\
\hline Grade 1 & Few vessels visible in the vicinity of the hilum \\
\hline Grade 2 & $\begin{array}{c}\text { Hilar and interlobar vessels visible in most of the } \\
\text { renal parenchyma }\end{array}$ \\
\hline Grade 3 & $\begin{array}{c}\text { Renal vessels identifiable until the arcuate arteries in } \\
\text { the entire field of view }\end{array}$ \\
\hline
\end{tabular}

oliguria or serum creatinine elevation within at least 3 days [21].

2.5. Statistical Analysis. Quantitative and qualitative data are expressed as median \pm standard deviation and numbers (percentages), respectively. The nonparametric test was used for categorical variables, and the $t$-test was for continuous variables between groups with and without PAKI. Counting data of patient outcome and SQC Doppler grade were analyzed by the $x^{2}$ test. Difference of RRI and SQC Doppler grade between the two operators was compared by using the Kendall test. Potential predictors were first tested by univariate logistic regression analysis, to determine the predictive ability of the RRI and SQC Doppler grade for the development of PAKI. A multivariate model was identified applying a p-entry and removed the value of $p$ less than 0.05 .

Using AKIN as the standard, receiver operating characteristic (ROC) analysis was performed on RRI and SQC Doppler grade to assess the efficiency of the two markers. All tests were two sided, and a probability value of less than 0.05 was considered statistically significant. SPSS 20.0 (SPSS Inc., IBM, USA) was used to analyze the data.

\section{Results}

3.1. Operator Consistence. The Kendall coefficient of RRI and SQC Doppler grade between the two operators was $0.827(n=14, P \leqq 0.001)$ and $0.693(n=14, P=0.027)$.

3.1.1. Primary Outcomes. Of a total of 67 patients during the study period, 21 (31.3\%) patients suffered from PAKI and 8 (11.9\%) patients required dialysis. Base characteristics were similar between the two groups (PAKI and without PAKI) except for preoperative $\mathrm{sCr}$ (Table 2). Intraoperative risk factors and postoperative influencing factors were similar between the two groups (PAKI and without PAKI) except for time of CPB and CRRT, length of stay in hospital, and length of stay in ICU (Table 3).

\subsubsection{Outcomes of RRI and SQC Doppler Grade}

(1) There is a significant difference in postoperative RRI in groups PAKI and without PAKI $(0.79 \pm 0.09$ vs. $0.72 \pm 0.06, P=0.002)$. There is also a significant difference in the SQC Doppler grade in between the two groups $\left(x^{2}=12.193, P=0.007\right)$ (Table 4$)$.

(2) Since 0 appears in the data of patients without PAKI, we combine SQC Doppler grade 0 and 1 to SQC Doppler grade 1 for univariate analysis. It shows that both RRI and SQC Doppler grade 2 were significant predictors of PAKI; besides, the other three significant predictors were length of stay in ICU, time of $\mathrm{CPB}$, and length of stay in hospital (Table 5).

(3) Similar to univariate regression analysis, we combine SQC Doppler grade 0 and 1 to SQC Doppler grade 1 for multivariate regression analysis. To determine whether RRI and SQC Doppler grade 2 were independent predictors of PAKI, the other three most significant variables in univariate analysis as confounders were analyzed for PAKI prediction in multivariate analysis, in addition to RRI and SQC Doppler grade. RRI remained a significant predictor of PAKI, independent of length of stay in ICU, time of $\mathrm{CPB}$, and length of stay in hospital. Similarly, the SQC Doppler grade is a predictor of PAKI independent of length of stay in ICU, time of CPB, and length of stay in hospital (Table 6).

(4) AUC of postoperative RRI to predict PAKI was 0.855 with cutoff value 0.725 (95\% CI, 0.74-0.96) (sensitivity $90.9 \%$ and specificity $71.1 \%$ ) (Figure $3(\mathrm{a})$ ). AUC of the SQC Doppler grade to predict PAKI was 0.642 (95\% CI, 0.49-0.79) with cutoff value grade 2 (sensitivity 50\% and specificity $73.3 \%$ ) (Figure 3(b)).

\section{Discussion}

This prospective study shows that both postoperative RRI and SQC Doppler grade are significant predictors of postoperative persistent AKI after AAAD surgery.

The two operators have good consistence at the RRI calculation and SQC Doppler grade evaluation, and the Kendall coefficient was $0.827(n=14, P \leqq 0.001)$ and 0.693 ( $n=14, P=0.027$ ), respectively. Close to the previous study [19], the technology with good reproducibility between the 2 operators avoided technique bias. The accuracy of RRI calculation and SQC Doppler grade evaluation still needs further study in larger scale samples and multiple centers.

The incidence of postoperative PAKI in our study was $31.3 \%$, and $11.9 \%$ patients required dialysis, which agrees with the previous study [22].

In this study, postoperative RRI was higher in PAKI patients than that in non-PAKI patients. Univariate and multivariate regression analysis certified that postoperative RRI was an independent predictor for developing of postoperative PAKI. Although preoperative sCr was different between groups with and without PAKI, but by the univariate regression analysis preoperative $\mathrm{sCr}$ is not a significant predictor, therefore not included in the multivariate analysis. The result of the AUC of postoperative RRI was 0.855 for predicting PAKI with a cutoff value of 0.725 (95\% CI, 0.49-0.79) (sensitivity $90.9 \%$, specificity $73.3 \%$ ). The cutoff value 0.725 was the same as that of our previous research [23], which indicates that RRI predicts postoperative AKI and has a fairly stable value in AAAD patients in our center. But it was higher than some studies $[24,25]$ and lower than other literatures $[26,27]$. We selected the average RRI in bilateral kidneys because of each kidney perhaps causing by different 


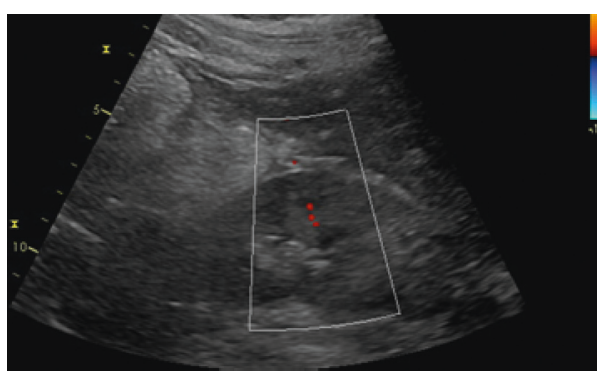

(a)

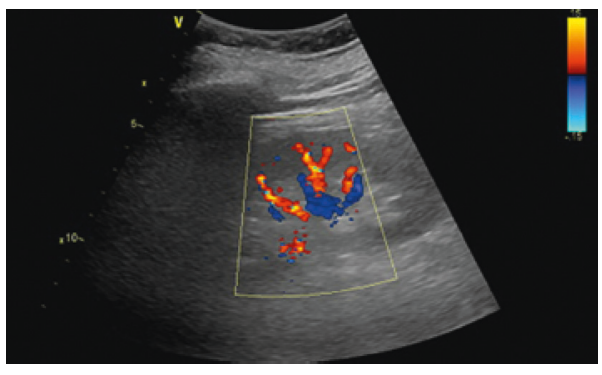

(c)

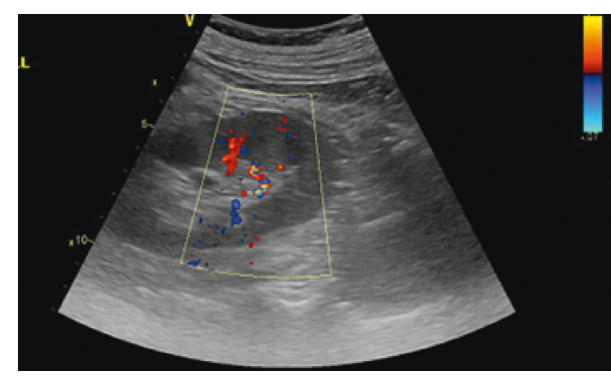

(b)

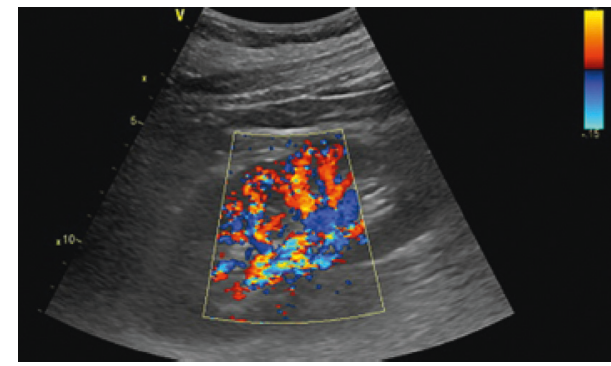

(d)

FIgURe 2: Semiquantitative color Doppler (SQC) grade. (a) Unidentifiable vessels were seen in the kidney; SQC grade was 0. (b) Vessels were seen in the renal hilum, but no vessels were seen in the parenchyma; SQC grade was 1. (c) Interlobar vessels were seen in most of the renal parenchyma; SQC grade was 2. (d) Arcuate arteries were seen in the entire field of view; SQC grade was 3.

TABle 2: Base characteristics between groups with PAKI and without PAKI.

\begin{tabular}{|c|c|c|c|c|}
\hline & All subjects with operation $(n=67)$ & Subjects without PAKI $(n=46)$ & Subjects with PAKI $(n=21)$ & $P$ value \\
\hline Male (\%) & $53(79.1)$ & $38(82.6)$ & $15(71.4)$ & 0.340 \\
\hline Age in years & $46.48 \pm 10.64$ & $45.61 \pm 10.76$ & $48.38 \pm 10.38$ & 0.360 \\
\hline BMI $\left(\mathrm{kg} / \mathrm{m}^{2}\right)$ & $27.85 \pm 6.35$ & $28.04 \pm 7.18$ & $27.45 \pm 4.11$ & 0.729 \\
\hline Hypertension (\%) & $58(86.5)$ & $39(84.7)$ & $19(90.4)$ & 0.709 \\
\hline Diabetes (\%) & $2(2.9)$ & $1(2.0)$ & $1(4.7)$ & 1.000 \\
\hline Smoking (\%) & $58(86.5)$ & $38(82.6)$ & $20(95.2)$ & 0.187 \\
\hline Drinking (\%) & $12(17.9)$ & $6(13.0)$ & $6(28.5)$ & 1.000 \\
\hline Preoperative $\mathrm{sCr}(\mu \mathrm{mol} / \mathrm{L})$ & $88.1 \pm 50.9$ & $78.5 \pm 26.6$ & $109.2 \pm 79.3$ & 0.021 \\
\hline MAP $(\mathrm{mm} \cdot \mathrm{Hg})$ & $83.3 \pm 12.4$ & $83.8 \pm 12.4$ & $83.5 \pm 12.5$ & 0.915 \\
\hline HR (beats $\min ^{-1}$ ) & $96 \pm 17$ & $97 \pm 17$ & $96 \pm 15$ & 0.950 \\
\hline $\mathrm{CVP}(\mathrm{mm} \cdot \mathrm{Hg})$ & $9.4 \pm 3.0$ & $9.0 \pm 2.0$ & $10.1 \pm 4.5$ & 0.155 \\
\hline $\mathrm{PaCO}_{2}(\mathrm{~mm} \cdot \mathrm{Hg})$ & $46.9 \pm 8.5$ & $46.5 \pm 7.6$ & $47.8 \pm 10.4$ & 0.559 \\
\hline $\mathrm{PaO}_{2}(\mathrm{~mm} \cdot \mathrm{Hg})$ & $158.8 \pm 274.0$ & $133.6 \pm 57.3$ & $213.9 \pm 448.3$ & 0.269 \\
\hline $\mathrm{EF}(\%)$ & $58.4 \pm 7.2$ & $59.4 \pm 6.6$ & $56.2 \pm 8.0$ & 0.086 \\
\hline
\end{tabular}

BMI: body mass index; MAP: mean arterial pressure; HR: heart rate; CVP: central venous pressure; EF: ejection fraction.

perfusion from the true or false lumen in AAAD patients. Furthermore, RRI cannot be evaluated in one of the bilateral kidneys because of poor kidney perfusion in 3 patients. It can result in deviations in the RRI values. Although multiple pathogenic pathways existed between RRI and AKI development [24, 28, 29], in conclusion our study certificated that postoperative RRI with cutoff value 0.725 was an independent predictor for the development of postoperative PAKI in AAAD patients.

In this study, patients below SQC Doppler grade 2 accounted for $71.4 \%$ in the PAKI group and $45.7 \%$ in the non-PAKI group. Univariate regression analysis certificated that SQC Doppler grade 2 was a significant predictor for postoperative PAKI; our study is the first to certify that SQC
Doppler grade was an independent predictor of postoperative PAKI by multivariate regression analysis. In this study, when we set the SQC Doppler grade less than 2 as a predictor for persistent AKI, AUC of SQC Doppler grade to predict PAKI was 0.642 (95\% CI, 0.49-0.79) with sensitivity $50 \%$ and specificity $73.3 \%$. The precision of SQC Doppler grade in predicting postoperative PAKI was poorer than postoperative RRI, which is in accordance with the recent study [30]. In critically ill patients, persistent AKI is considered to be the result of acute tubular necrosis which is caused by initially reduced renal perfusion [31]. The finding can help solving the problem of rapid, flexible, inexpensive, and noninvasive in patients after surgery at the intensive care unit. 
TABLE 3: Intraoperative and postoperative characteristics between groups with PAKI and without PAKI.

\begin{tabular}{|c|c|c|c|c|c|}
\hline & & $\begin{array}{l}\text { All subjects } \\
(n=67)\end{array}$ & $\begin{array}{l}\text { Subjects without PAKI } \\
\qquad(n=46)\end{array}$ & $\begin{array}{l}\text { Subjects with PAKI } \\
\quad(n=21)\end{array}$ & $\begin{array}{c}P \\
\text { value }\end{array}$ \\
\hline & Time of CPB (min) & $205 \pm 43$ & $198 \pm 43$ & $221 \pm 40$ & 0.037 \\
\hline & $\begin{array}{l}\text { Time of aortic cross clamping } \\
\text { (min) }\end{array}$ & $123 \pm 33$ & $125 \pm 160$ & $120 \pm 23$ & 0.059 \\
\hline & Time of DHCA (min) & $25.0 \pm 11.3$ & $23.3 \pm 8.8$ & $28.9 \pm 14.8$ & 0.889 \\
\hline & Urine output (ml) & $1848 \pm 1065$ & $1910 \pm 958$ & $1714 \pm 1285$ & 0.489 \\
\hline \multicolumn{6}{|l|}{$\begin{array}{l}\text { Intraoperative } \\
\text { drugs }\end{array}$} \\
\hline & Dopamine & $3.57 \pm 2.55$ & $3.48 \pm 2.50$ & $3.76 \pm 2.60$ & 0.676 \\
\hline & Adrenaline & $0.025 \pm 0.020$ & $0.024 \pm 0.021$ & $0.026 \pm 0.018$ & 0.802 \\
\hline \multicolumn{6}{|l|}{ Outcomes } \\
\hline & Postoperative sCr $(\mu \mathrm{mol} / \mathrm{L})$ & $140.32 \pm 129.62$ & $116.72 \pm 138.56$ & $188.61 \pm 94.55$ & \\
\hline & CRRT $(\%)$ & $8(11.9)$ & $2(4.3)$ & $6(28.6)$ & 0.009 \\
\hline & Length of stay in hospital (days) & $14.5 \pm 8.4$ & $13.4 \pm 1.8$ & $16.8 \pm 9.4$ & 0.003 \\
\hline & Length of stay in ICU (days) & $4.7 \pm 3.7$ & $3.8 \pm 3.5$ & $6.6 \pm 3.5$ & 0.009 \\
\hline
\end{tabular}

CPB: cardiopulmonary bypass; DHCA: deep hypothermic circulatory arrest; CRRT: continuous renal replacement therapy.

TABLE 4: Comparison of RRI and semiquantitative color Doppler grade between groups with PAKI and without PAKI.

\begin{tabular}{lccc}
\hline & All subjects $(n=67)$ & Subjects without PAKI $(n=46)$ & Subjects with PAKI $(n=21)$ \\
\hline$R R I$ & & & \\
& $0.75 \pm 0.08$ & $0.72 \pm 0.06$ & $0.79 \pm 0.09$ \\
\hline Semiquantitative color Doppler grade & & & \\
0 & $3(4.4 \%)$ & $0(0 \%)$ & $3(14.3 \%)$ \\
1 & $6(8.9 \%)$ & $1(2.2 \%)$ & $5(23.8 \%)$ \\
2 & $27(40.3 \%)$ & $20(29.8 \%)$ & $7(33.3 \%)$ \\
3 & $31(46.2 \%)$ & $25(54.3 \%)$ & $6(28.6 \%)$ \\
& & & 0.007 \\
\hline
\end{tabular}

TABLE 5: Univariate regression analysis for PAKI.

\begin{tabular}{|c|c|c|c|}
\hline & OR & OR $(95 \% \mathrm{CI})$ & $P$-value \\
\hline Male & 0.377 & $0.114-1.245$ & 0.109 \\
\hline Age & 1.025 & $0.976-1.078$ & 0.322 \\
\hline BMI & 0.997 & $0.919-1.082$ & 0.944 \\
\hline Hypertension & 1.842 & $0.350-9.709$ & 0.471 \\
\hline Preoperative $s \mathrm{Cr}(\mu \mathrm{mol} / \mathrm{L})$ & 1.106 & $0.999-1.033$ & 0.063 \\
\hline MAP $(\mathrm{mm} \cdot \mathrm{Hg})$ & 0.957 & $0.956-1.039$ & 0.869 \\
\hline HR (beats $\min ^{-1}$ ) & 0.995 & $0.964-1.026$ & 0.747 \\
\hline $\mathrm{CVP}(\mathrm{mm} \cdot \mathrm{Hg})$ & 1.138 & $0.941-1.377$ & 0.182 \\
\hline $\mathrm{PaCO}_{2}(\mathrm{~mm} \cdot \mathrm{Hg})$ & 1.018 & $0.951-1.081$ & 0.562 \\
\hline $\mathrm{PaO}_{2}(\mathrm{~mm} \cdot \mathrm{Hg})$ & 1.001 & $0.999-1.103$ & 0.377 \\
\hline $\mathrm{EF}(\%)$ & 0.958 & $0.878-1.045$ & 0.330 \\
\hline Time of CPB (min) & 1.015 & $1.002-1.028$ & 0.021 \\
\hline Time of aortic cross clamping (min) & 1.000 & $0.996-1.004$ & 0.972 \\
\hline Time of DHCA (min) & 1.037 & $0.991-1.085$ & 0.112 \\
\hline Dopamine & 1.061 & $0.865-1.301$ & 0.571 \\
\hline Adrenaline & 2.241 & $0.001-1.755$ & 0.950 \\
\hline Urine output (ml) & 1.000 & $0.999-1.000$ & 0.439 \\
\hline CRRT & 0.049 & $0.006-0.429$ & 0.006 \\
\hline Length of stay in hospital (days) & 1.085 & $1.010-1.166$ & 0.025 \\
\hline Length of stay in ICU (days) & 1.670 & $1.256-2.221$ & $<0.001$ \\
\hline RRI & 6.553 & $2.454-17.503$ & $<0.001$ \\
\hline \multicolumn{4}{|l|}{ SQC Doppler grade } \\
\hline 2 & 27.429 & $2.912-258.387$ & 0.004 \\
\hline 1 & 1.200 & $0.360-4.000$ & 0.767 \\
\hline
\end{tabular}


TABLE 6: Multivariate regression analysis for PAKI.

\begin{tabular}{|c|c|c|c|c|c|c|}
\hline & OR & OR $(95 \% \mathrm{CI})$ & $P$ value & OR & OR $(95 \% \mathrm{CI})$ & $P$ value \\
\hline Time of CPB (min) & 1.006 & $0.989-1.023$ & 0.515 & 1.004 & $0.991-1.023$ & 0.414 \\
\hline Length of stay in hospital (days) & 0.962 & $0.861-1.073$ & 0.486 & 0.957 & $0.848-1.079$ & 0.471 \\
\hline Length of stay in ICU (days) & 1.512 & $1.075-2.218$ & 0.018 & 1.733 & $1.204-2.493$ & 0.003 \\
\hline RRI & 4.110 & $1.396-12.013$ & 0.010 & & & \\
\hline SQC Doppler grade 2 & & & & 19.380 & $1.406-267.208$ & 0.027 \\
\hline 1 & & & & 1.798 & $0.395-8.197$ & 0.448 \\
\hline
\end{tabular}

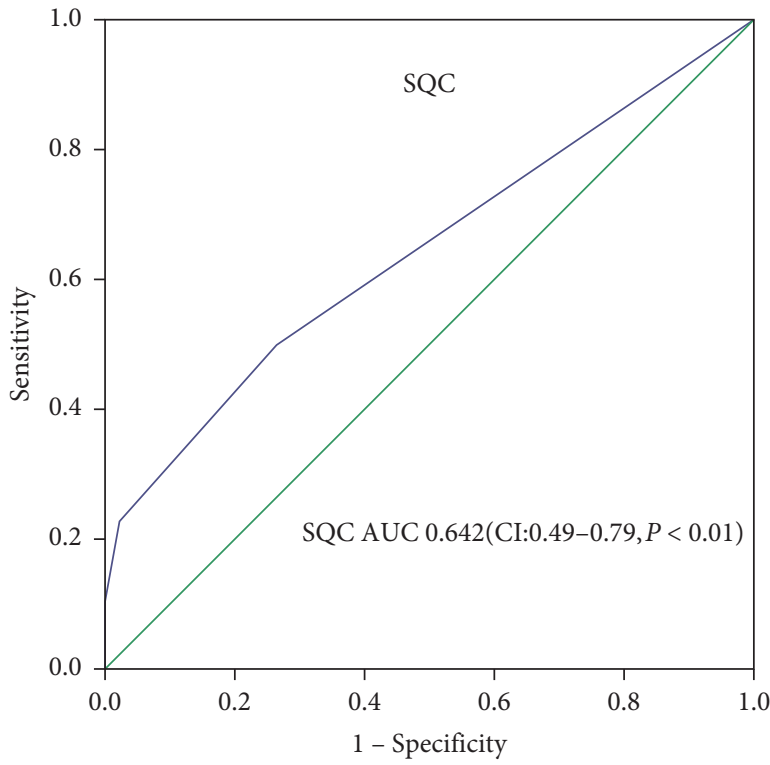

(a)

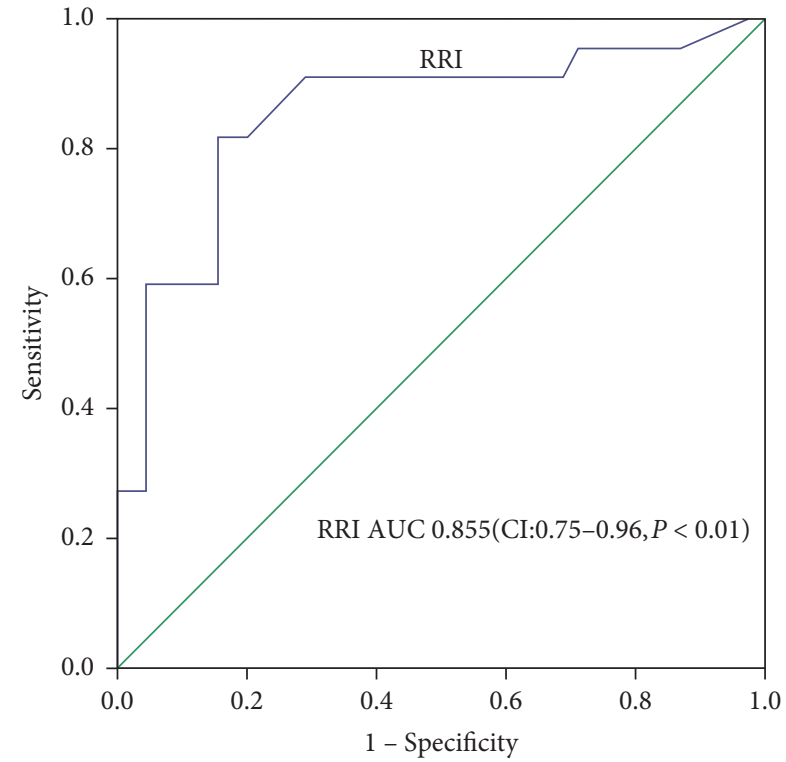

(b)

FIGURE 3: Prediction of postoperative persistent acute renal injury with ROC curves using postoperative RRI (a) and SQC Doppler grade (b).

In conclusion, both postoperative RRI and SQC Doppler grade are independent predictors for PAKI after surgery in AAAD patients. Moreover, both postoperative RRI and SQC Doppler grade can be obtained rapidly by bedside ultrasound, which is an important diagnostic tool in the case of clinical conditions that might impair kidney function [32].

There was no significant difference in demographic and clinical information between the PAKI and without PAKI groups except for preoperative sCr. However, preoperative sCr was not an independent predictor of postoperative AKI by univariate and multivariate analysis in this research. Moreover, to avoid intrarenal hemodynamic changes causing bias, we measured RRI and evaluated the kidney perfusion by ultrasound in the period with mechanical ventilation, supine, during a narrow range of $\mathrm{PaCO}_{2}$. Because the level of breathing, body position and $\mathrm{PaCO}_{2}$ may affect RRI measurement. [33].

We have two strengths: (1) our study is the first to certify SQC Doppler grade as an independent predictor of postoperative PAKI by multivariate regression analysis; (2) the cutoff value 0.725 of postoperative RRI was the same as that of our previous research, which indicates it will have good applicability in AAAD patients in our center.
There are some limitations in our study. (1) The blood flow in the kidney was not easily observed in some obese patients or severe AKI patients. Bias in judgment of the results may exist. (2) The length of the six months study period and the limitation of the small sample, so the statistical results have a large confidence interval. (3) This was a single-center and single-disease study. A further study with wider range of applications, larger sample, and multiple centers is required.

\section{Conclusion}

Both postoperative RRI and SQC Doppler grade are independent predictors for PAKI after surgery in AAAD patients.

Both postoperative RRI and SQC Doppler grade can be obtained rapidly by bedside ultrasound, which is a good tool for early prediction for postoperative PAKI.

\section{Data Availability}

The data used to support the findings of this study are available from the corresponding author upon request. 


\section{Conflicts of Interest}

All authors declare that they have no conflicts of interest.

\section{Authors' Contributions}

Huai Qin and Zhanming Fan conceived and designed the experiments; Huai Qin and Yaqiong Liperformed the experiments; Huai Qin, Nan Zhang, and TiezhuWang analyzed the data; Huai Qin and Nan Zhang contributed reagents/ materials/analysis tools; and all authors were involved in writing the manuscript.

\section{Acknowledgments}

This work was supported by the funding from the Department of Cardiovascular Intensive Care, Beijing Anzhen Hospital, Capital Medical University, and Beijing Institute of Heart, Lung, and Blood Vessel Diseases.

\section{References}

[1] J. A. Tumlin, K. W. Finkel, P. T. Murray, J. Samuels, G. Cotsonis, and A. D. Shaw, "Fenoldopam mesylate in early acute tubular necrosis: a randomized, double-blind, placebocontrolled clinical trial," American Journal of Kidney Diseases, vol. 46, no. 1, pp. 26-34, 2005.

[2] R. Hirschberg, J. Kopple, P. Lipsett et al., "Multicenter clinical trial of recombinant human insulin-like growth factor I in patients with acute renal failure," Kidney International, vol. 55 , no. 6, pp. 2423-2432, 1999.

[3] I. C. Vermeulen Windsant, M. G. Snoeijs, S. J. Hanssen et al., "Hemolysis is associated with acute kidney injury during major aortic surgery," Kidney International, vol. 77, no. 10, pp. 913-920, 2010.

[4] J. L. Koyner, M. R. Bennett, E. M. Worcester et al., "Urinary cystatin $\mathrm{C}$ as an early biomarker of acute kidney injury following adult cardiothoracic surgery," Kidney International, vol. 74, no. 8, pp. 1059-1069, 2008.

[5] A. Ristikankare, R. Pöyhiä, A. Kuitunen et al., "Serum cystatin $\mathrm{C}$ in elderly cardiac surgery patients," The Annals of Thoracic Surgery, vol. 89, no. 3, pp. 689-694, 2010.

[6] A. Gaipov, Y. Solak, K. Turkmen et al., "Serum uric acid may predict development of progressive acute kidney injury after open heart surgery," Renal Failure, vol. 37, no. 1, pp. 96-102, 2015.

[7] J. Ho, N. Tangri, P. Komenda et al., "Urinary, plasma, and serum biomarkers' utility for predicting acute kidney injury associated with cardiac surgery in adults: a meta-analysis," American Journal of Kidney Diseases, vol. 66, no. 6, pp. 993-1005, 2015.

[8] A. Harrois, N. Grillot, S. Figueiredo, and J. Duranteau, "Acute kidney injury is associated with a decrease in cortical renal perfusion during septic shock," Critical Care, vol. 22, no. 1, p. 161, 2018.

[9] F. G. Meinel, C. N. De Cecco, U. J. Schoepf, and R. Katzberg, "Contrast-induced acute kidney injury: definition, epidemiology, and outcome," BioMed Research International, vol. 2014, no. 2, Article ID 859328, 6 pages, 2014.

[10] P. Mccullough, "Outcomes of contrast-induced nephropathy: experience in patients undergoing cardiovascular intervention," Catheterization and Cardiovascular Interventions, vol. 67, no. 3, pp. 335-343, 2010.
[11] R. J. Earlam, "The radio-isotope renogram in the non-functioning kidney," British Journal of Urology, vol. 38, no. 3, pp. 288-293, 1966.

[12] N. Lerolle, E. Guérot, C. Faisy, C. Bornstain, J.-L. Diehl, and J.-Y. Fagon, "Renal failure in septic shock: predictive value of Doppler-based renal arterial resistive index," Intensive Care Medicine, vol. 32, no. 10, pp. 1553-1559, 2006.

[13] M. T. Wybraniec, M. Boentowicz-Wikarek, J. Chudek et al., "Pre-procedural renal resistive index accurately predicts contrast-induced acute kidney injury in patients with preserved renal function submitted to coronary angiography," The International Journal of Cardiovascular Imaging, vol. 33, no. 5, pp. 595-604, 2017.

[14] P. Marty, F. Ferre, F. Labaste et al., "The doppler renal resistive index for early detection of acute kidney injury after hip fracture," Anaesthesia Critical Care \& Pain Medicine, vol. 35, no. 6, pp. 377-382, 2016.

[15] H. Huang, J. Zhou, and J. Chen, "Biomarkers for early diagnosis of acute kidney injury: current progress and clinical prospects," Current Protein \& Peptide Science, vol. 18, no. 12, 2017.

[16] M. E. Murphy and M. E. Tublin, "Understanding the Doppler RI: impact of renal arterial distensibility on the RI in a hydronephrotic ex vivo rabbit kidney model," Journal of Ultrasound in Medicine, vol. 19, no. 5, pp. 303-314, 2000.

[17] R. O. Bude and J. M. Rubin, "Relationship between the resistive index and vascular compliance and resistance," $R a$ diology, vol. 211, no. 2, pp. 411-417, 1999.

[18] M. Darmon, F. Schortgen, F. Vargas et al., "Diagnostic accuracy of Doppler renal resistive index for reversibility of acute kidney injury in critically ill patients," Intensive Care Medicine, vol. 37, no. 1, pp. 68-76, 2011.

[19] D. Schnell, M. Reynaud, M. Venot et al., "Resistive index or color-Doppler semi-quantitative evaluation of renal perfusion by inexperienced physicians: results of a pilot study," Minerva Anestesiologica, vol. 80, no. 12, pp. 1273-1281, 2014.

[20] M. E. Wolfswinkel, L. C. Koopmans, D. A. Hesselink et al., "Neutrophil gelatinase-associated lipocalin (NGAL) predicts the occurrence of malaria-induced acute kidney injury," Malarian Journal, vol. 15, no. 1, p. 464, 2016.

[21] R. L. Mehta, J. A. Kellum, S. V. Shah et al., "Acute kidney injury network: report of an initiative to improve outcomes in acute kidney injury," Critical Care, vol. 11, no. 2, p. R31, 2007.

[22] H. Zhao, X. Pan, Z. Gong et al., "Risk factors for acute kidney injury in overweight patients with acute type A aortic dissection: a retrospective study," Journal of Thoracic Disease, vol. 7, no. 8, pp. 1385-1390, 2015.

[23] H.-B. Wu, H. Qin, W.-G. Ma, H.-L. Zhao, J. Zheng, and L.-Z. Sun, "Can renal resistive index predict acute kidney injury after acute type A aortic dissection repair?," The Annals of Thoracic Surgery, vol. 104, no. 5, pp. 1583-1589, 2017.

[24] P. Marty, S. Szatjnic, F. Ferre et al., "Doppler renal resistive index for early detection of acute kidney injury after major orthopaedic surgery," European Journal of Anaesthesiology, vol. 32, no. 1, pp. 37-43, 2015.

[25] P.-G. Guinot, E. Bernard, O. Abou Arab et al., "Doppler-based renal resistive index can assess progression of acute kidney injury in patients undergoing cardiac surgery," Journal of Cardiothoracic and Vascular Anesthesia, vol. 27, no. 5, pp. 890-896, 2013.

[26] G. Bossard, P. Bourgoin, J. J. Corbeau, J. Huntzinger, and L. Beydon, "Early detection of postoperative acute kidney injury by Doppler renal resistive index in cardiac surgery with 
cardiopulmonary bypass," British Journal of Anaesthesia, vol. 107, no. 6, pp. 891-898, 2011.

[27] S. Ninet, D. Schnell, A. Dewitte, F. Zeni, F. Meziani, and M. Darmon, "Doppler-based renal resistive index for prediction of renal dysfunction reversibility: a systematic review and meta-analysis," Journal of Critical Care, vol. 30, no. 3, pp. 629-635, 2015.

[28] A. Dewitte, J. Coquin, B. Meyssignac et al., "Doppler resistive index to reflect regulation of renal vascular tone during sepsis and acute kidney injury," Critical Care, vol. 16, no. 5, p. R165, 2012.

[29] N. Lameire, W. Van Biesen, and R. Vanholder, "Acute renal problems in the critically ill cancer patient," Current Opinion in Critical Care, vol. 14, no. 6, pp. 635-646, 2008.

[30] J. Hashimoto and S. Ito, "Central pulse pressure and aortic stiffness determine renal hemodynamics: pathophysiological implication for microalbuminuria in hypertension," Hypertension, vol. 58, no. 5, pp. 839-846, 2011.

[31] M. Darmon, A. Bourmaud, M. Reynaud et al., "Performance of Doppler-based resistive index and semi-quantitative renal perfusion in predicting persistent AKI: results of a prospective multicenter study," Intensive Care Medicine, vol. 44, no. 11, pp. 1904-1913, 2018.

[32] A. Granata, P. Di Nicolò, V. R. Scarfia et al., "Renal transplantation parenchymal complications: what Doppler ultrasound can and cannot do," Journal of Ultrasound, vol. 18, no. 2, pp. 109-116, 2015.

[33] T. Ko, M. Higashitani, A. Sato et al., "Impact of acute kidney injury on early to long-term outcomes in patients who underwent surgery for type A acute aortic dissection," The American Journal of Cardiology, vol. 116, no. 3, pp. 463-468, 2015. 


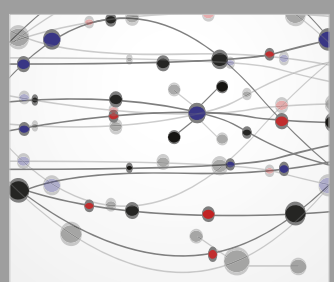

The Scientific World Journal
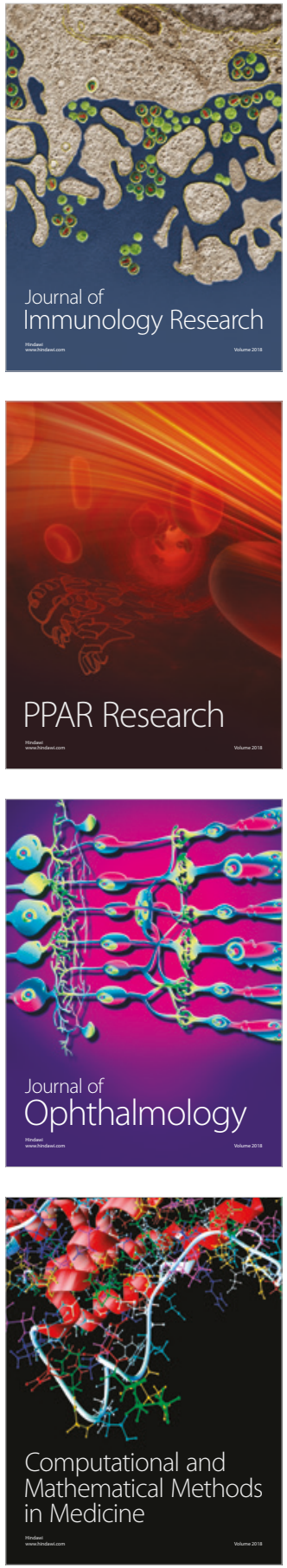

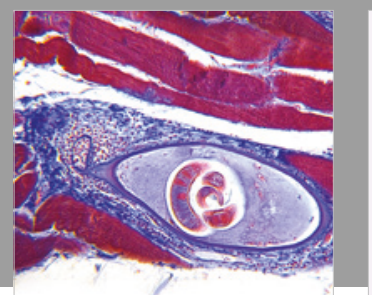

Gastroenterology Research and Practice

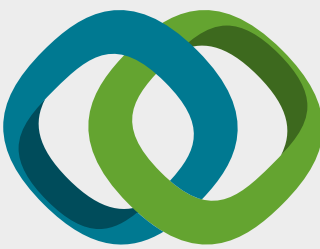

\section{Hindawi}

Submit your manuscripts at

www.hindawi.com
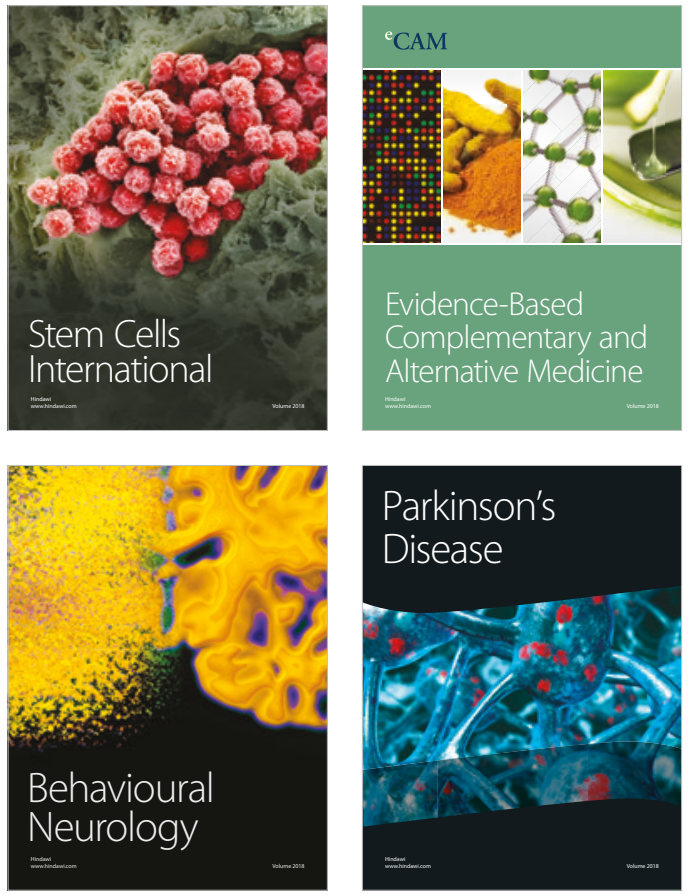

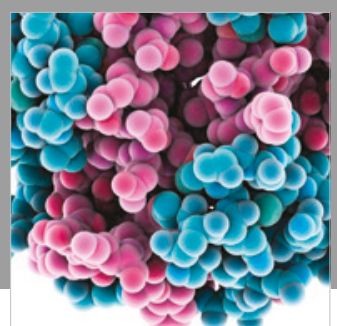

ournal of

Diabetes Research

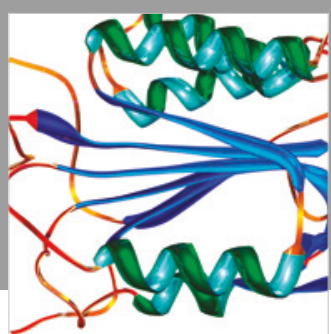

Disease Markers
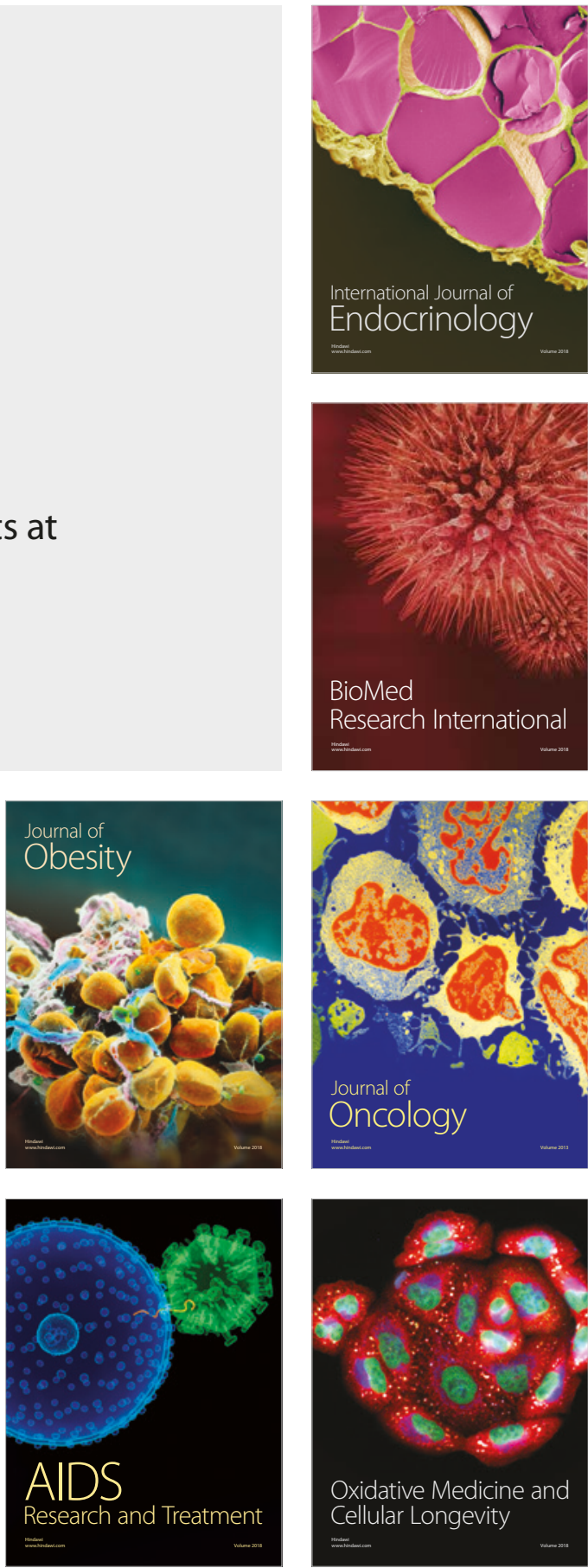